Radiologe 2010 · 50:653

DOI 10.1007/s00117-010-2039-0

Online publiziert: 15. Juli 2010

(c) Springer-Verlag 2010

\author{
M. Puderbach · S. Delorme \\ Abt. Radiologie (E010), Deutsches Krebsforschungszentrum (DKFZ) Heidelberg
}

\title{
Bronchialkarzinom: Neues aus Diagnostik und Therapie
}

Wie auch bei anderen Tumoren bleibt das N-Staging schwierig, denn die Unterscheidung reaktiver von kleineren metastatisch befallenen Lymphknoten ist allein aufgrund der Größe praktisch nicht möglich. Aus diesem Grund ist das präoperative Staging des Bronchialkarzinoms derzeit die einzige Indikation, bei der sich der Gemeinsame Bundesausschuss zu einer Zulassung der PET hat erweichen lassen. In der Tat sind die Bedingungen für die PET im Thorax besonders günstig, weil sich Tumoren wie befallene Lymphknoten gut vor dem niedrigen Hintergrundsignal abheben (s. Beitrag von Clemens Kratochwil).

Von allen Schnittbildverfahren hat heute der Ultraschall im Nahfeld die höchste Ortsauflösung. Mit endobronchialen Schallsonden ist eine Beurteilung der Struktur von Lymphknoten möglich. Weiterhin ermöglicht dieses Verfahren die präzise ultraschallgeführte Biopsie peri- oder extrabronchialer Prozesse mit einer in der Hand des geübten Untersuchers niedrigen Rate falsch-negativer Biopsate und leistet somit einen entscheidenden Beitrag zur Stadieneinteilung des Bronchialkarzinoms (s. Beitrag von Daniela Gompelmann).

Die MRT ist längst dem Stadium entwachsen, da man zeigte, dass man in einer Stunde Untersuchungszeit das Gleiche darstellen kann wie mit der CT in einer Minute. Sie kann inzwischen viel mehr, ihr Potenzial liegt zum einen in der morphologischen Bildgebung mit hohem Gewebekontrast zur Differenzierung von Tumor und Atelektase und zur besseren Visualisierung infiltrierter Strukturen, aber auch in der funktionellen Bildgebung (Perfusions-, Bewegungsmessung). Hieraus ergeben sich die Möglichkeiten zur besseren Einschätzung der Prognose und der Erfassung von Therapieeffekten (s. Beitrag von Christian Hintze). Bereits heute ist die MRT eine wichtige Ergänzung und z. T. auch Alternative zur CT.

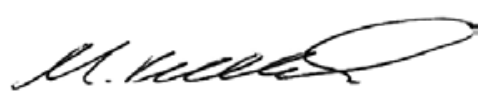

Dr. Michael Puderbach
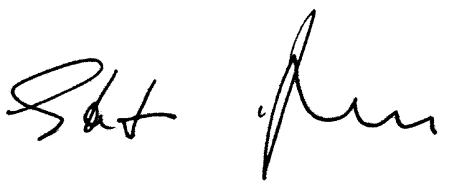

Prof. Dr. Stefan Delorme 